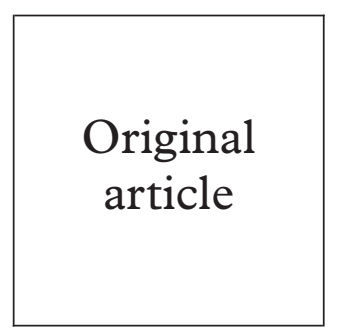

\title{
Syphilis serology and HIV infection in Harare, Zimbabwe
}

Lovemore Gwanzura, Ahmed Latif, Mary Bassett, Rhoderick Machekano, David A Katzenstein, Peter R Mason

Objective: To determine the reliability of serological tests in detecting syphilis in a factory worker cohort and examine the impact of concurrent HIV infection on serological tests for syphilis. Method: Reactions to non-treponemal and treponemal antigens were tested using sera from a cohort of 3401 factory workers in Harare, Zimbabwe. The participants consented to regular testing for syphilis, by VDRL, and HIV using two ELISAs. All sera from men who were VDRL positive, and a random sample of VDRL negative sera, were tested by RPR, TPHA, and where appropriate FTA-Abs. From the results, men were defined as having no syphilis, active syphilis, incident syphilis, historic syphilis, or giving biological false positive reactions.

Results: 709 sera were examined from 580 men. There were 78 cases of active syphilis in the cohort, giving a prevalence of $2.3 \%$, and the seroincidence was 0.25 per 100 person years of follow up. The prevalence of HIV in the cohort was $19.8 \%$. There was a strong association between syphilis, whether active, incident or historic, and HIV seropositivity. With both HIV positive and negative sera the negative predictive values of VDRL and RPR were $>99.9 \%$ while the positive predictive value for VDRL (30\%) was lower than for RPR (39\%). Biological false positive reactions were detected in $0.5 \%$ of the cohort, with in most cases a transient rise in VDRL titres up to $<1 / 16$. Higher false positive titres occurred in five men, each of whom was HIV positive.

Conclusions: The VDRL is reliable in detecting possible cases of syphilis even in a community with a high prevalence of heterosexually transmitted HIV. There is need, however, for confirmatory tests. The prevalence of syphilis in this cohort is very low in comparison with other countries in southern Africa, but is consistent with recent data from Harare. Despite a strong association between syphilis and HIV it was clear that syphilis could not be counted as a major factor fuelling the HIV epidemic in Zimbabwe.

(Sex Transm Inf 1999;75:426-430)

Keywords: syphilis; serodiagnosis; HIV; Treponema pallidum; Zimbabwe

\section{Introduction}

A significant epidemiological association between infection with Treponema pallidum (syphilis) and infection with the human immunodeficiency virus (HIV) has been demonstrated in many studies in Africa $^{1-3}$ and elsewhere. ${ }^{4}$ While both infections show similarity in the mode of transmission, ulcerative genital lesions contain large numbers of both CD4 and CD8 lymphocytes, and so may be a site of both HIV shedding and invasion. ${ }^{56}$ Interventions directed towards reducing the incidence of syphilis and other sexually transmissible infections are regarded as important strategies in HIV control, ${ }^{78}$ and information on the epidemiology of syphilis is necessary for the design of such programmes.

Atypical clinical presentation of syphilis has been described in patients with $\mathrm{HIV}^{4-11}$ and abnormalities are more pronounced in patients with low CD4 cell counts. ${ }^{4}$ Because immune responses to $T$ pallidum are primarily cell mediated, the loss of cellular immunity may intensify the ability of the organism to multiply in a variety of tissues, resulting in persistent genital ulceration and an increased likelihood of symptoms of secondary syphilis, gummatous lesions, and rapid progression to neurosyphilis. ${ }^{11-13}$
The laboratory diagnosis of syphilis relies on serological assays ${ }^{14}{ }^{15}$ which include nontreponemal screening tests and treponemal confirmatory tests. Non-treponemal tests such as VDRL (Venereal Disease Reference Laboratory) and RPR (rapid plasma reagin) detect antibodies to a cardiolipin-lecithin-cholesterol antigen, and they are positive in $>99 \%$ of cases of secondary syphilis. They are, however, much less sensitive in detecting early, latent, or late syphilis. Specific treponemal tests include haemagglutination, fluorescent antibody, and enzyme immunoassays, with TPHA ( $T$ pallidum haemagglutination assay), and FTA-Abs (fluorescent treponemal antibody using absorbed sera) being the most frequently used of these. Biological false positive (BFP) reactions are defined as a rise in titre to non-treponemal reagin antigens while specific treponemal tests remain negative. Transient BFP may occur in a variety of acute viral and other infections and chronic BFP reactions may be detected in non-infectious conditions such as lupus erythematosus and malignancy and in injecting drug users (IDU). The BFP rate may be as high as $50 \%$ in selected populations, though it is expected to be of the order of $1-2 \%$ in a healthy population. ${ }^{14-16}$ The reliability of treponemal antibody tests in syphilis is regarded as being very high, with $<1 \%$ false 
positives, even among sera from patients with lupus or from IDU. ${ }^{15}$ The low cost and simplicity of non-treponemal serology tests, however, means they are frequently used for screening large numbers of sera. Positive sera are then subjected to specific treponemal tests to distinguish BFP reactions from true syphilis infection.

The reliability of non-treponemal tests in detecting syphilis in HIV positive individuals is uncertain. A high prevalence of both BFP and false negative reactions has been recorded ${ }^{15} 17$ while recent studies have indicated that at least some apparent BFP may in fact represent false negative treponemal serology. ${ }^{19}$ Most of the studies on syphilis serology in HIV positives have used populations that include IDU and, as noted above, such people are at high risk of giving chronic BFP reactions.

In order to examine the impact of HIV infection on serological responses to syphilis and to provide data on epidemiology of syphilis, we examined sera collected from factory workers in Harare, Zimbabwe under the Zimbabwe AIDS Prevention Project (ZAPP).

\section{Materials and methods \\ ZAPP COHORT}

The ZAPP enrolled a rolling cohort of 3401, mostly male, factory workers in Harare, Zimbabwe, between 1993 and 1996. The participants gave consent to regular serological testing for HIV, syphilis, and hepatitis B infection, and provided demographic and behavioural information. The details of the cohort and risk factors for HIV seropositivity have been given elsewhere. ${ }^{20}$ The protocol was approved by the Medical Research Council of Zimbabwe and by institutional review boards of the University of Zimbabwe and Stanford University.

\section{SERUM SPECIMENS}

Clotted blood samples were collected at enrolment into the cohort, and thereafter blood was collected at about 4 monthly intervals during the programme. Blood samples were centrifuged, and sera were stored in aliquots of about $0.5 \mathrm{ml}$. One aliquot was used for serological testing at the time of sample preparation, and others were stored at $-20^{\circ} \mathrm{C}$ for follow up studies. Aliquots were stored in sequence, using laboratory, ZAPP identification, and date labels to ensure accurate and efficient retrieval.

\section{SEROLOGICAL TESTING}

Initial screening of sera for antibodies to HIV-1 and HIV-2 and syphilis was carried out at the National Blood Transfusion Service (NBTS) laboratories in Harare, using two separate HIV ELISAs (Abbott Labs, USA; Behring, Germany), and a modified VDRL assay using carbon antigen (Cambridge Biotech, UK). The assays were carried out using a standard operating procedure, and the results were stored on the ZAPP database. The NBTS is a national testing and training centre for identification of safe blood, and has a high standard of laboratory expertise. They subscribe to the Zimbabwe National Quality Assurance Programme (ZINQAP) for medical laboratories.

\section{SERUM SELECTION}

For the purposes of this study, the ZAPP database was screened to identify the following serum samples:

(a) One aliquot from every bleed for members of the cohort who gave a positive VDRL reaction at any time, whether at enrolment or during follow up. This ensured that we identified all cases of active syphilis, prevalent and incident, in the study cohort.

(b) One enrolment serum aliquot from members of the cohort who were consistently VDRL negative throughout the programme. The sera were used to determine antibodies to specific treponemal antigens in VDRL and RPR negative sera, and they were selected from the ZAPP database to include about equal numbers of HIV positives and HIV negatives. Financial constraints meant that the whole cohort sample could not be examined in this way.

(c) Aliquots of follow up sera from all members of the cohort who, although VDRL seronegative, were positive by any other test for antibodies to syphilis as described below. Sera that were VDRL negative but positive by a treponemal test may have resulted from either acute syphilis infection or old inactive syphilis. These were distinguished on the basis of examination of the follow up sera.

SYPHILIS SEROLOGY AND DIAGNOSIS

The sera collected in this study were retested for antibodies to syphilis using the following protocol. The previously obtained VDRL result was recorded and the sera were then tested using RPR (Immutrep RPR, Omega Diagnostics). If the RPR result was concordant with the VDRL result, the sera were tested by TPHA only (Immutrep TPHA, Omega Diagnostics). If there was a difference between the results of the two non-treponemal tests, or if there was a difference in results using enrolment and follow up sera (that is, seroconversion or transient BFP) the sera were tested with both TPHA and FTA-Abs (Wellcome Labs). In each case the test was carried out under the supervision of a qualified medical technologist and the manufacturer's methods and interpretation of results were closely followed. For RPR sera were tested undiluted, and for TPHA sera were tested at a dilution of $1 / 80$ in the diluent supplied by the manufacturer. In addition to the kit controls, known positive and negative sera from other studies in Zimbabwe were used as internal controls. A randomly selected proportion of the sera $(n=85,12 \%)$ was also tested independently by the laboratory of the Biomedical Research and Training Institute as an external quality control. The BRTI laboratory also subscribes to the ZINQAP in serology. There were no differences in test results.

On the basis of these results, members of the cohort were identified as having: 
Table 1 Non-treponemal serologies in the factory worker cohort

\begin{tabular}{|c|c|c|c|c|c|c|c|}
\hline & \multicolumn{3}{|c|}{ Syphilis status } & & \multicolumn{3}{|c|}{ Syphilis status } \\
\hline & Active & Non & & & Active & Nor & \\
\hline \multicolumn{4}{|l|}{ VDRL } & \multicolumn{4}{|l|}{$\mathrm{RPR}$} \\
\hline Pos & 157 & 19 & 176 & Pos & 160 & 4 & 164 \\
\hline \multirow[t]{2}{*}{ Neg } & 9 & 475 & 484 & Neg & 6 & 490 & 496 \\
\hline & 166 & 494 & $660^{\star}$ & & 166 & 494 & $660^{\star}$ \\
\hline \multicolumn{2}{|c|}{ VDRL sensitivity } & \multicolumn{2}{|c|}{$157 / 166(94.6)$} & \multicolumn{2}{|c|}{ RPR sensitivity } & \multicolumn{2}{|c|}{$160 / 166(96.4 \%)$} \\
\hline \multicolumn{2}{|c|}{ VDRL specificity } & \multicolumn{2}{|c|}{$475 / 494(96.2 \%)$} & \multicolumn{2}{|c|}{ RPR specificity } & \multicolumn{2}{|c|}{$490 / 494(99.2 \%)$} \\
\hline \multicolumn{8}{|c|}{$\begin{array}{l}\text { Applying these data to the cohort, with a syphilis prevalence of } 2.3 \% \text {, the positive predictive } \\
\text { values (PPV), and negative predictive values (NPV) are }\end{array}$} \\
\hline \multicolumn{4}{|c|}{ VDRL-NPV $=3144 / 3147(>99.9 \%)$} & \multicolumn{4}{|c|}{ RPR-NPV = 3203/3204 (>99.9\%) } \\
\hline \multicolumn{4}{|c|}{ VDRL-PPV $=75 / 254(29.5 \%)$} & \multicolumn{4}{|c|}{ RPR-PPV $=77 / 197(39.1 \%)$} \\
\hline
\end{tabular}

${ }^{\star}$ Sera from participants with incident or historic syphilis were excluded.

(a) No syphilis if all tests were negative. If only a non-treponemal test was positive, the result was regarded as a BFP reaction. In such cases the VDRL and RPR titres were determined using doubling dilutions of serum as described by the manufacturers

(b) Active syphilis if the enrolment serum was positive for at least one non-treponemal and one treponemal test

(c) Incident syphilis if the enrolment serum showed no syphilis while any of the follow up sera showed active syphilis, as defined in (b) above

(d) Historic syphilis if both enrolment and follow up sera gave a positive reaction in at least one treponemal test, and a negative reaction with both non-treponemal tests.

These definitions, rather than the classic terminology of primary, latent, and late syphilis, were used because they are based on serological criteria only rather than the more usual combination of both serological and clinical findings. All participants with active or incident syphilis were offered treatment with a single dose of 2.4 million units of benzathine penicillin (incident cases) or with three doses each of 2.4 million units of benzathine penicillin administered at weekly intervals (prevalent cases). This treatment is in accordance with national guidelines. Men identified with syphilis were encouraged to bring their sexual partner(s) for testing and treatment.

DATA ANALYSIS

The sensitivity and specificity of VDRL and RPR for syphilis screening was determined for both HIV positive and HIV negative sera. The sensitivity and specificity data were then applied to the cohort as a whole.

\section{Results}

RELIABILITY OF VDRL AS A SCREEN FOR SYPHILIS A total of 709 sera were examined from 580 participants. There were 483 (83\%) participants with no evidence of syphilis, 78 (13\%) with serological evidence of active syphilis, 13 $(2 \%)$ with incident syphilis, and six $(1 \%)$ with historic syphilis. Because the sampling method collected all sera that were VDRL positive, and the negative predictive value of VDRL is very high (see below), the prevalence of active syphilis in the ZAPP cohort was 78/3401 $(2.3 \%)$. The true prevalence of historic syphilis may be higher than estimated here because we only examined sera from about $12 \%$ of the whole cohort using the treponemal tests that detect past infections. The prevalence of HIV at enrolment was $49 \%(38 / 78)$ in men with active syphilis, $62 \%(8 / 13)$ in men with incident syphilis, and $67 \%(4 / 6)$ in men with historic syphilis. The prevalence of HIV in men with no evidence of syphilis $(42 \%, 195 / 467)$ was skewed by the selection process which specifically required about equal numbers of HIV positive and HIV negative sera. The enrolment HIV prevalence in the cohort as a whole was $19.8 \%$. HIV seropositivity was highly associated with serological evidence of syphilis, whether active, incident, or historic, with 50/97 (51.5\%) such men being HIV positive compared with $610 / 3304(18.5 \%)$ men who had no evidence of syphilis $\left(\chi^{2} 5.9, \mathrm{p}<0.0001\right)$.

The diagnosis of no syphilis and active syphilis reached above was used as the "gold standard" to determine the sensitivity and specificity of screening tests for syphilis. This analysis included results from syphilis screening of all sera including BFPs. Cases of incident and historic syphilis were, however, excluded from this analysis, since these were by definition VDRL negative on enrolment. The results (table 1) indicate the RPR to have a slightly higher sensitivity and specificity than the VDRL. There was no difference in sensitivity or specificity when analysed separately by HIV serostatus (data not shown). Since these tests detect antibodies to a disease state, rather than a specific organism, the positive and negative predictive values are of greater importance. Assuming the syphilis prevalence in the whole cohort to be $2.3 \%$, the VDRL had a positive predictive value of only $29.5 \% \quad(75 / 254)$ compared with $39.1 \%$ (77/197) for RPR. The negative predictive values for both tests were $>99.9 \%$ under these conditions.

If TPHA had been used as a screen, active syphilis would have been detected in 164/166 $(99 \%)$ men who had active syphilis by our definition, the other two sera being positive by FTA-Abs but not by TPHA. Each TPHA positive serum that was tested was also positive by FTA-Abs. With eight $(62 \%)$ incident participants the TPHA became positive at the same time as FTA-Abs, while in five $(38 \%)$ cases the FTA-Abs became positive before the TPHA. With four (31\%) participants, all HIV positive, the VDRL was positive before TPHA, and in two cases also before FTA-Abs.

BIOLOGICAL FALSE POSITIVE REACTIONS

Twenty serum samples from 16 participants gave non-treponemal false positive reactions, 16 with VDRL only, one with RPR only, and three with both. Of these 20 BFP, 15 were at low VDRL titre ( $1 / 8$ or less) while three were at a titre of $1 / 16$ and two were at a titre of $1 / 32$. These last five sera were all from HIV seropositive men. Sera obtained subsequent to the BFP sample were available from 14 participants, and in all except one case both VDRL and RPR as well as treponemal tests were negative. In one case, the serum was positive by both VDRL and RPR, but negative by treponemal tests at enrolment, and the subsequent sample was VDRL positive but RPR 
negative. The next and each subsequent sample were negative for syphilis by all serological tests. Nine of the 16 BFP samples were from HIV positive men.

\section{Discussion}

The primary method for detecting suspected active syphilis in the cohort was a positive VDRL reaction. Because VDRL had a very high negative predictive value (>99.9\%), we can be reasonably certain that all suspect cases in the cohort were in fact identified, though specific treponemal tests were necessary to establish true infections. The syphilis serology data from the subsample can therefore be applied to the cohort as a whole with a high degree of confidence. On this basis, the enrolment prevalence of syphilis in this cohort was $2.3 \%$. This is substantially lower than Tanzania (6\%), Ethiopia (9\%), and Mozambique $(12 \%)^{1-3}$ though the population in our cohort may not be directly comparable. The incidence of syphilis in this community was also very low $(0.25$ new cases of syphilis per 100 person years). During the past decade a decline in the numbers of antenatal cases of syphilis has been noted by the city of Harare. ${ }^{21}$ Even among female sex workers, who may be assumed to be at particularly high risk of infection, the seroprevalence of syphilis in 1995 was only $3.4 \% .^{22}$ The data we present here are consistent with these records. The reasons why syphilis should be an uncommon infection in Zimbabwe are not clear, but extensive use of penicillin for the treatment of many infections, plus condom promotion and safe sex behaviour programmes may be important factors.

Despite this low prevalence and incidence of syphilis there was a strong epidemiological association between syphilis and HIV. Men who were HIV seropositive had a threefold higher risk of having serological evidence of active syphilis, and a similar trend was noted with incident syphilis. A history of genital ulceration has been shown to be a significant risk factor in HIV seroconversion in this cohort, ${ }^{23}$ but because the overall prevalence of syphilis was so low other causes of genital ulceration, such as HSV-2, may be more significant. ${ }^{24}$

We found 16 donors whose sera had false positive VDRL or RPR reactions. Because our selection procedure identified all VDRL positive sera, these data can be applied to the cohort as a whole, giving a VDRL BFP rate of about $0.5 \%$. This is similar to the BFP rate detected in samples from the general population in Jamaica, ${ }^{16}$ but is lower than in studies using populations with large numbers of IDU. ${ }^{11}$ Infection with HIV, and possibly with sexually transmitted viral infections, such as herpes virus, may induce polyclonal $\mathrm{B}$ cell activation, ${ }^{25}$ perhaps resulting in transient rises in levels of "reagin" antibodies. The titres of RPR reactive antibody were usually low, with more than half the sera having a titre of $<1 / 8$, though it should be noted that all of the sera with a titre $>1 / 16$ were from HIV seropositive men. We found four syphilis seroconverters, all of whom were
HIV seropositive, who became VDRL positive before FTA-Abs and/or TPHA. This suggests that the production of antibodies to treponemal antigen may be delayed in HIV positive individuals, and that some BFP may in fact represent false negative treponemal serology as has been noted elsewhere. ${ }^{19}$

Of importance also is our finding that the negative predictive value of a VDRL is very high even when using sera from HIV positive men. This would validate the use of VDRL in screening blood donors for serological evidence of syphilis even in communities where HIV infection is prevalent.

We thank ZAPP staff for assistance in blood and data collections, and the National Blood Transfusion Service for identifying the sera used The ZAPP is supported by US Public Health Service Award No A1 33868-02

Contributors: L Gwanzura was responsible for the selection Contributors: L Gwanzura was responsible for the selection
and laboratory testing of samples and assisted with data analysis and write up; A Latif and D A Katzenstein are principal clinica researchers in ZAPP and assisted in study design and analysis $M$ Basset is director of ZAPP in Harare and assisted in writing the article; R Machkano supplied statistical advice in serum selection and analysis of data; and P R Mason supervised the study design and analysis, and was responsible for writing the article.

1 Cossa HA, Glod S, Vaz RG, et al. Syphilis and HIV infection among displaced pregnant women in rural Mozambique. among displaced pregnant wom

2 Assefa A, Rahlenbeck S, Molla K, et al. Seroprevalence of HIV-1 and syphilis antibodies in blood donors in Gonder, Ethiopia. F Acq Immun Def Syndr 1994;7:1282-5.

3 Killewo JZ, Sandstrom A, Bredberg Raden U, et al. Prevalence and incidence of syphilis and its association with HIV-1 infection in a population-based study in the Kagera region of Tanzania. Int 7 STD AIDS 1994;5:42431.

4 Schoffer H, Imhof M, Thoma-Groper E, et al. Active syphilis in HIV infection: a multicentre retrospective study. Genitourin Med 1996;72:176-81

5 Ghys PD, Fransen K, Diallo MO, et al. The association between cervicovaginal HIV shedding, sexually transmitted diseases and immunosuppression in female sex workers in diseases and immunosuppression in temale sex

6 Van Voorhis WC, Barrett LK, Nasio JM, et al. Lesions of primary and secondary syphilis contain activated cytolytic $T$ cells. Infect Immun 1996;64:1048-50.

7 Robinson NJ, Mulder DW, Auvert B, et al. Proportion of HIV infections attributable to other sexually transmitted diseases in a rural Ugandan population:simulation model estimates. Int f Epidemiol 1997;26:180-9.

8 Wasserheit JN. Epidemiological synergy. Interrelationships between human immunodeficiency virus infection and other sexually transmitted diseases. Sex Transm Dis 1992;19:61-77.

9 Musher DM, Hamill RJ, Baugh RE. Effect of human immunodeficiency virus (HIV) infection on the course of syphilis and the response to treatment. Ann Intern Med 1990; 11:872-88.

10 Hicks CB. Syphilis and HIV infection. Dermatol Clin 1991, 9:493-501.

11 Hutchinson CM, Hook EW, Shepherd M, et al. Altered clinical presentation of early syphilis in patients with human immunodeficiency virus infection. Ann Intern Med 1994;121:94-100.

12 Johns DR, Tierney M, Felsentein D. Alteration in the natural history of neurosyphilis by concurrent infection with the human immunodeficiency virus. N Engl f Med 1987;316: 1569-72.

13 Katz DA, Berger R, Duncan RL. Neurosyphilis: a comparative study of the effects of infection with human immunodeficiency virus. Arch Neurol 1993;50:243-9.

14 Larsen SA, Hunter EF, Creighton ET. Diagnostic testing for syphilis. In: Holmes KK, Mardh PA, Sparling PF, Weissner syphilis. In: Holmes KK, Mardh PA, Sparling PF, Weissner PJ, eds. Sexually transmitted diseases.
Hill, Part VII, Chapter 75:927-34.

15 Larsen SA, Steiner BM, Rudolph AH. Laboratory diagnosis and interpretation of tests for syphilis. Clin Microbiol Rev 1995;8:1-21

16 Smikle MF, James O B'L, Prabhakar P. Biological false positive serological tests for syphilis in the Jamaican population. Genitourin Med 1990;66:76-8.

17 Augenbraun MH, DeHovitz JA, Feldman J, et al. Biologic false-positive syphilis test results for women infected with human immunodeficiency virus. Clin Infect Dis 1994;19: 1040-4.

18 Nandwani R, Evans DT. Are you sure it's syphilis? A review of false positive serology. Int $\mathcal{F}$ STD AIDS 1995;6:241-8.

19 Erbelding EJ, Vlahov D, Nelson KE, et al. Syphilis serology in human immunodeficiency virus infection: evidence for false-negative fluorescent treponemal testing. F Infect Dis 1997;176:1397-400. 
20 Bassett MT, McFarland WC, Ray S, et al. Risk factors for HIV infection at enrollment in an urban male factor cohort in Harare, Zimbabwe. F Acq Immun Def Syndr Hum Retrovir 1996;13:287-93.

21 Annual Reports of the Department of Health Services, City of Harare, Harare, Zimbabwe, 1987-1997.

22 Mason PR, Ray S, Maposhere C, et al. Use of female and male condoms by commercial sex workers: impact on STD transmission. IXth International Conference on AIDS and STD in Africa. Kampala, Uganda 1995. Abstract No TuC614.
23 Mbizvo MT, Latif AS, Machekano R, et al. HIV seroconversion among factory workers in Harare: who is getting newly infected? Centr Afr F Med 1997;43:135-9.

24 Gwanzura L, McFarland W, Alexander D'A, et al. Association between human immunodeficiency virus and herpes simplex virus type 2 seropositivity among male factory workers in Zimbabwe. F Infect Dis 1998;177:481-4.

25 Lane HC, Masur H, Edgar LC, et al. Abnormalities of B-cell activation and immunoregulation in patients with the acquired immunodeficiency syndrome. $N$ Engl $f$ Med 1983;309:453-8.

\section{Joint Meeting of MSSVD and the ASTDA}

3-7 May 2000, Baltimore Marriott Inner Harbor Hotel, Baltimore, Maryland, USA

To mark the unique nature of the millennial year, for the first time in its 78 year history the Medical Society for the Study of Venereal Diseases will hold its Spring Meeting jointly with the American Sexually Transmitted Disease Association (President, Professor Julius Schacter). Our local host will be Professor Jonathan Zenilman of Johns Hopkins Medical School. The scientific programme will consist of plenary lectures and round table discussions, delivered by world authorities. There will also be oral and poster presentations of original work.

Further mailings will follow to MSSVD and ASTDA members. People who do not belong to either of these organisations and who would like to receive further information should contact: Dr Keith Radcliffe, Honorary Assistant Secretary, MSSVD (fax: +44 (0) 121-237 5729; email: k.w.radcliffe@bham.ac.uk). 